Chapter 2

\title{
SRAP Molecular Marker Technology in Plant Science
}

\author{
Genyi Li, Peter B. E. McVetty and Carlos F. Quiros \\ Additional information is available at the end of the chapter \\ http://dx.doi.org/10.5772/54511
}

\section{Introduction}

Molecular markers are commonly used in genetic diversity analysis, genetic map construction, gene mapping and cloning, and marker assisted selection in plant breeding. Based on detection procedure, most molecular marker technologies can be classified into hybridization-based or PCR-based systems. Restriction fragment length polymorphism (RFLP) is the first hybridization-based molecular marker system that was intensively used at the beginning of the molecular biology era in life science while hybridization-based marker methods such as microarrays and diversity array technology (DArT) are used currently to detect single nucleotide polymorphisms (SNP). In contrast, many PCR-based molecular marker detection methods have been developed. For example, amplified fragment length polymorphism (AFLP), random amplified polymorphic DNA (RAPD), simple sequence repeats (SSR) and sequence related amplified polymorphism (SRAP), inter-simple sequence repeat (ISSR), sequence tagged site (STS), and sequence characterized amplification region (SCAR), are commonly used in genomic analysis (Jones et al., 2009).

There are advantages and limitations for all molecular marker detection methods. In particular, RFLP probes can be shared in related species so RFLP is advantageous over other molecular markers in comparative genomics. However, the detection procedure in RFLP is complicated and costly. Additionally, RFLP is not easily automated to analyze thousands of individuals for marker assisted selection. AFLP is a commonly used molecular marker system since it can detect multiple genetic loci in a genome. On the other hand, there are many steps in the AFLP detection procedure, which limits its application in marker assisted selection when thousands of individual DNA samples need to be analyzed in a short time. SSRs often have a high level of polymorphism in plant genomes and are commonly used in most genomic applications. Since SSR technology only detects sequence repeats, the number of SSRs in a genome is relatively limited compared with numerous SNPs. RAPD is easily per- 
formed in one round of PCR, however, a low level of reproducibility of RAPD amplification limits its wide use in genomic analysis.

As next generation sequencing (NGS) technologies dramatically increase capacity and throughput of DNA sequencing, whole genome sequencing of many plant species has been accomplished and most economically important crop species such as rice, maize, soybean, sorghum, potato, tomato and Chinese cabbage have been fully sequenced. Although it is still challenging to use NGS for assembling a whole complex genome such as barley and wheat, there are thousands of SNPs identified in NGS that can be used to develop molecular markers in species with complex genomes. Furthermore, NGS is directly used in SNP discovery and a few dozen genotypes can be sequenced simultaneously to assemble ultradense genetic maps. Additionally, different strategies are used to produce partial genomes that can be used to directly sequence SNPs using next generation technologies.

\section{SRAP technology}

We developed and published information on the SRAP marker system in 2001 (Li and Quiros). The original thinking was to simplify the AFLP detection procedure and increase throughput and improve reproducibility compared to RAPD. To produce a simple detection procedure, we skipped restriction enzyme digestion and ligation of target DNA fragments and adapters in the AFLP detection protocol. We designed SRAP primers in sizes similar to those in AFLP, but ran one round of PCR instead of two rounds in AFLP. To detect multiple loci with a pair of SRAP primers, we designed a special PCR running program $\left(94^{\circ} \mathrm{C}\right.$ for 1 min, $35^{\circ} \mathrm{C}$ for $1 \mathrm{~min}$ and $72^{\circ} \mathrm{C}$ for $1 \mathrm{~min}$ for the first 5 cycles and followed by 30 cycles at the raised annealing temperature of $50^{\circ} \mathrm{C}$ ). At the beginning of $\mathrm{PCR}$, the $35^{\circ} \mathrm{C}$ annealing temperature allowed SRAP primers to anneal to multiple loci in target DNA so that the multiple loci were amplified to produce a profile that is similar to that in AFLP. Similar to AFLP, most SRAP markers are dominant while most SNPs and SSRs are co-dominant. Compared with RAPD, SRAP used a pair of primers with 16 to 22 nucleotides instead of 10-mer short primers in RAPD, which gives SRAP a big advantage over RAPD so one SRAP primer can combine with unlimited number of other primers. Although SRAP PCR starts at $35^{\circ} \mathrm{C}$ annealing temperature in the first five cycles, the larger sizes of SRAP primers allowed the increase of annealing temperature to $50^{\circ} \mathrm{C}$ in the following cycles, which significantly improves the reproducibility in SRAP. In contrast, a low level of reproducibility in RAPD is a limitation factor. In addition, SRAP primers can be fluorescently labelled and combined with unlabeled SRAP primers so SRAP PCR products can be separated in capillary instruments such as ABI genetic analyzers.

In general, there is a difference of GC content between gene coding sequences and other sequences in plant genomes. We used this difference to design two sets of SRAP primers. The forward primers contained a GGCC cassette closing the $3^{\prime}$ end of SRAP primers that might preferentially anneal to the GC-rich regions while the reverse SRAP primer set was incorporated with an AATT cassette that would preferentially anneal SRAP primers to introns and 
gene spacers so that SRAP could preferentially amplify gene-rich regions in a genome. After sequencing SRAP fragments and constructing a SRAP genetic map in B. oleracea, it was found that SRAP indeed amplified more sequences from genes and more SRAP markers fell into chromosome arm regions and produced fewer markers in the centromeres that were filled with AFLP markers.

There is wide flexibility in the design of SRAP primers. After testing the primers we used for gene cloning, we found that most of these primers worked well in SRAP amplification. In the construction of an ultra-dense genetic map in B. napus, we used 12 fluorescently labeled forward primers and 442 unlabeled primers to assemble a genetic map with 13,351 molecular markers (Sun et al., 2007). Based on the SRAP markers on the genetic map, the efficiency of each labeled primer was checked by counting the average numbers of SRAP markers produced by individual labeled primers. Some labeled SRAP primers such as FC1, BG23 and SA7 produced more than ten polymorphic loci while EM2 and DC1 produced less than six polymorphic loci. If we checked individual SRAP primer combinations, we found that there was a big difference in the numbers of polymorphic loci detected by individual SRAP primers. Efficient SRAP primer combinations produced over 20 polymorphic loci while less efficient SRAP primer combinations produced one to three mapped loci. Actually, we tested over a thousand SRAP primers in B. napus and B. rapa and found that most primers produced good profiles with over 30 strong bands (unpublished data). After testing a large set of SRAP primer combinations, we selected a set of SRAP primer combinations that showed the best performance in SRAP amplification in different accessions and populations in various Brassica species. These SRAP primer combinations serve as a standard set and are routinely used in genetic map construction, mapping of quantitative loci (QTL) and gene cloning in our lab. Therefore, if SRAP is frequently used in a lab, it is worthwhile to select a set of SRAP primer combinations which will enhance the effectiveness and efficiency of SRAP marker detection.

To enhance the capacity and effectiveness of SRAP technology, we combined SRAP with Illumina's Solexa sequencing to directly integrate genetic loci on the B. rapa genetic map based on paired-end Solexa sequences (Li et al., 2011). To achieve this objective, we used two rounds of PCR to prepare SRAP products that were pooled and sequenced with Illlumina's Solexa sequencing. The first round of PCR produced SRAP fragments using individual DNA samples from a mapping population with the same set of SRAP primer combinations while the second round was used to tail the SRAP PCR products that allowed identifying the original DNA for producing paired-end Solexa sequences. After Illumina's Solexa sequencing, paired-end sequences were sorted using the sequences of tag primers and the numbers of unique paired-end sequences from each DNA samples were obtained. These numbers represent Solexa sequence frequencies in each DNA sample which was used to integrate Solexa sequences onto the SRAP genetic map. To integrate paired-end sequences on the genetic map, we adopted a QTL mapping strategy by using Windows QTL Cartographer software 2.5. When a paired-end sequence was found to have only one significant LOD score in a bin of the genetic map, this sequence was assigned into this bin. In total, 1737 unique paired-end sequences representing the same number of genetic loci were integrated 
on the genetic map. Eventually, we constructed a high density B. rapa genetic map consisting of 1,737 paired-end Solexa sequences, 9,177 SRAP markers and 46 SSR markers.

\section{Genetic map construction}

Genetic maps are extensively used in gene mapping, QTL mapping and assembly of whole genome sequence. High density molecular markers in genetic maps are advantageous and necessary in most applications. The detection of multiple loci in a SRAP PCR reaction can be automated through fluorescently labelled SRAP primers so it is feasible to construct a high density genetic map using SRAP technology.

In B. napus, we used a five-color fluorescent dye set including '6-FAM', 'VIC', 'Pet', 'NED' and 'LIZ' to perform SRAP fragment analysis with the ABI genetic analyzer (Sun et al., 2007). 'LIZ' was used as the internal standard while the other four fluorescent dyes were used to label SRAP primers and combined with unlabeled primers. After obtaining SRAP products with four fluorescently labeled and four unlabeled primers, all the products from four SRAP primer combinations were pooled to increase the detection throughput by four fold. We used 1,634 SRAP primer combinations selected from 12 labeled and 442 unlabeled SRAP primers to produce 13,472 SRAP markers. Together with 79 SSR markers, we assembled currently the most saturated genetic map in $B$. napus.

Using cDNA-SRAP technology, we first constructed a transcriptome map based on B. oleracea cDNAs obtained from leaf tissue (Li et al., 2003). In cDNA-SRAP, one step PCR allows the amplification of single strand cDNAs after the first strand CDNA is synthesized using reverse transcriptase. Since most cDNA-SRAP markers come from differences in gene sequence, these markers are considered to be functional markers. SRAP products can be easily isolated from polyacrylamide gels for sequencing so we sequenced 190 fragments that corresponded to 190 polymorphic loci from cDNA-SRAP. Through analysis of sequence similarity, 169 out of 190 cDNA marker sequences were homologous to genes reported in Arabidopsis, which allowed the identification of extensive colinearity between the two genomes according to the gene-for-gene alignment. Later, we developed over 1,000 SRAP markers using genomic DNA from the same mapping population and assembled these SRAP markers from both genomic DNA and cDNA samples on the same genetic map (Gao et al., 2007). In addition, we integrated 10 SCAR markers using sequences of genes with known functions in the biosynthesis of glucosinolates and inflorescence architecture, and one SCAR marker flanking a resistance gene to downy mildew.

SRAP technology can be combined with other markers to construct genetic maps. For example, Yu et al., (2007) constructed a high-density genetic map in a cultivated allotetraploid cotton population using SSR, SRAP, AFLP, and target region amplification polymorphism (TRAP). This high density cotton genetic map consists of 697 SSR, 171 TRAP, 129 SRAP, 98 AFLP, and two morphological markers, covering a genetic distance of 4,536.7 cM with the average genetic distance of $4.1 \mathrm{cM}$ per marker. Gulsen et al., (2010) reported a new citrus linkage map using SRAP, RAPD, SSR, ISSR, peroxidase gene polymorphism (POGP), resist- 
ant gene analog (RGA), and a morphological marker, Alternaria brown spot resistance gene. In total, they assembled 385 SRAP, 97 RAPD, 95 SSR, 18 ISSR, 12 POGP, and 2 RGA markers on the citrus genetic map.

In the Cucurbitaceae family, Yeboah et al., (2007) constructed genetic maps in cucumber using SRAP and ISSR markers. They developed pseudo-testcross F1 segregating populations from a cross between two diploid parents and constructed male and female parental genetic maps separately with 164 SSR and 108 SRAP markers. More recently, Zhang et al., (2012) constructed a high density consensus genetic map in an inter-subspecific mapping population in cucumber. The consensus map contained over a thousand molecular markers including 1,152 SSR, 192 SRAP, 21 SCAR and one STS. In another cucurbit species, Levi et al., (2006) constructed an extended genetic map for watermelon using five PCR-based molecular markers SRAP, AFLP, SSR, ISSR and RAPD. As suggested by the authors, low polymorphism is often observed in watermelon cultivars, combining several marker systems is necessary to construct a high density genetic map covering the whole genome.

SRAP markers have been used to construct genetic maps in a wide range of plant species. In Dendrobium plants that are used as Chinese herbs, Xue et al., (2010) constructed two genetic maps in two Dendrobium species, D. officinale and D. hercoglossum with a double pseudotestcross strategy using SRAP and RAPD methods. In root plants, Chen et al., (2010) constructed a genetic map in an $\mathrm{F}_{1}$ population derived from an interspecific cross in cassava by combining AFLP, SSR, SRAP and expressed sequence tag (EST)-SSR markers. In total, they assembled 355 markers into 18 linkage groups covering a genetic distance of 1,707.9 cM, which served as a foundation for QTL mapping in this species. In grass species, Xie et al., (2011) used SSR and SRAP markers to construct two genetic maps of male and female parental lines respectively in diploid orchardgrass (Dactylis glomerata L.) using a pseudo-test cross strategy. In total, they assembled 164 SSR markers and 108 SRAP markers on these two genetic maps. In a fruit tree, Luohanguo (Siraitia grosvenorii C. Jeffrey), Liu et al., (2011) used SRAP and ISSR markers to assemble a genetic map consisting of 170 SRAP markers and 29 ISSRs in 25 linkage groups. In a fiber crop, Chen et al., (2011) used SRAP, ISSR and RAPD markers to construct a genetic map in Kenaf (Hibiscus cannabinus L.) that is one of the most economically important fiber crops globally.

\section{QTL mapping}

A common application of genetic maps is QTL mapping of complex traits. Since QTL are often underpinned by multiple genes in a genome, it is difficult to tag QTL using procedures for tagging Mendelian loci. In general, if a complex trait is changed into a simple Mendelian trait and the underlying QTL is Mendelized, many strategies are available to map and clone the Mendelized genes. In fact, most cloned QTL have been accomplished through such a Mendelized strategy by developing near-isogenic lines (NILs). However, most QTL are not easily Mendelized, so it is necessary to construct genetic maps first and then perform QTL mapping. 
In canola, we used SRAP and SSR to construct a genetic map in a doubled haploid (DH) line population that was developed from a synthetic yellow-seeded line and a conventional canola cultivar through microspore culture (Chen et al., 2009). Data for three complex traits including days to flowering, oil content and seed yield at three locations for three years were collected and used in QTL mapping. For oil content, 27 QTL on 14 linkage groups and for seed yield, 18 QTL on 11 linkage groups were identified while days to flowering was suggested to be controlled by a single genetic locus in this mapping population. In rapeseed, Chen et al., (2007) used 208 SSR and 189 SRAP markers to construct a genetic map for a DH line population and performed QTL mapping of yield-related traits in B. napus. They also developed a fixed immortalized population from randomly permutated intermating of these $\mathrm{DH}$ lines. They collected data for six yield-related traits, plant height, height of lowest primary effective branch, length of main inflorescence, silique length, number of primary branches and silique density. After QTL mapping in the DH line and immortalized populations, they identified 29 common QTL between the two populations, suggesting that there are some chromosomal regions containing QTL for multiple traits. In another QTL mapping report, Fu et al., (2007) constructed a genetic map in B. napus to map gene loci controlling the yellow seeded trait. They developed 420 SSR, RAPD and SRAP markers and assembled two genetic maps, of which one contained 26 linkage groups and another which had 20 linkage groups. After QTL analysis, they identified 19 QTL with one common in the two mapping populations. Further analysis allowed them to identify the collinear genomic region of chromosome 5 in Arabidopsis.

In cotton, Lin et al., (2005) developed a mapping population by crossing G. hirsutum and G. barbadense and performed QTL mapping with a genetic map constructed with SRAP, SSR and RAPD markers. Using 437 SRAP, 107 RAPD and 205 SSR markers, they constructed a genetic map with 566 markers assembled into 41 linkage groups, of which 28 were assigned to the corresponding known chromosomes. This genetic map was used to perform QTL mapping for fiber traits. In total, 13 QTL for fibre traits including two QTL for fibre strength, four QTL for fibre length and seven QTL for micronaire value were identified, of which six QTL were assigned into the A-subgenome, another six QTL into the D-subgenome while one QTL was not assigned. Three QTL for micronaire value were identified to cluster on linkage group 1, suggesting that the flanking molecular markers of these three QTL might be useful in marker-assisted selection for this trait.

Similarly, Zhang et al., (2009b) reported on QTL mapping in cotton using SRAP and other markers. They assembled a genetic map containing 509 SSR, 58 intron targeted intron/exon splice junction (IT-ISJ), 29 SRAP and 8 morphological loci in 60 linkage groups. Among these 60 linkage groups, 54 were assigned into 26 chromosomes. This genetic map was used to identify QTL for fiber quality traits in five environments. In total, thirteen QTL including four QTL for fiber length, two QTL for fiber strength, two QTL for fiber fineness, three QTL for fiber length uniformity, and two QTL for fiber elongation were identified. Eleven out of 13 QTL were assigned into the A-subgenome and other two QTL, into the D-subgenome.

In chrysanthemum (Dendranthema morifolium), Zhang et al., (2011b) performed QTL mapping of inflorescence-related traits using SRAP markers. They constructed two genetic maps 
in a $F_{1}$ segregating population using a double pseudo-testcross mapping strategy. With 500 SRAP primer combinations, they produced 896 polymorphic loci and assembled 333 SRAP markers into 57 linkage groups on one genetic map, 342 SRAP markers into 55 linkage groups on the second genetic map. The results indicated that the distribution of these SRAP markers on these two genetic maps was quite uniform. Using these two genetic maps, they mapped 12 QTL for three inflorescence traits, of which each four QTL underpinned specified flower diameter, ray floret layer number, and ray floret length, respectively.

In radish, $\mathrm{Xu}$ et al., (2012) recently constructed a genetic map with 592 molecular markers including 287 SRAP, 135 RAPD, 78 SSR, 49 ISSR, 29 randomly amplified microsatellite polymorphism (RAMP), and 14 resistant gene analogs (RGA). They used this genetic map to analyze QTL that controlled root cadmium accumulation. They mapped four QTL on linkage groups 1. 4. 6 and 9. The QTL on linkage group 9 was a major one that accounted for $48.64 \%$ of phenotypic variance, suggesting that this QTL might be applied for marker assisted selection to improve radish root quality by reducing cadmium concentration.

\section{Gene tagging and cloning}

SRAP technology has several merits for gene tagging. Since SRAP detection uses unlimited primer combinations and there are multiple loci detected in a single SRAP PCR reaction, SRAP technology is advantageous over other molecular marker systems for gene tagging. After many genetic loci in a genome are screened quickly, closely linked SRAP markers to a trait of interest can be identified easily. We intensively used SRAP to perform gene tagging and cloning in Brassica species and worked on several economically important traits such as yellow-seeded canola and rapeseed, disease resistance and glucosinolates.

Yellow-seeded oilseeds in Brassica species are suggested to be related to high oil content so it is worthwhile to characterize the genes controlling seed coat color. Using SRAP technology, we cloned and characterized a gene controlling seed coat color and plant hairiness traits in B. rapa (Zhang et al., 2009a). We used 1,100 SRAP primer combinations to screen pooled DNA from yellow-seeded and black-seeded individuals based on the bulk segregant analysis (BSA) strategy and found 48 SRAP primer combinations that produced polymorphic loci in the pooled DNA samples from yellow-seeded and black-seeded individuals. Then we tested more pooled DNA and identified 13 SRAP markers that were linked to the gene of interest. Sequencing these SRAP markers allowed the identification of a chromosomal region that was further used to develop new SCAR markers. With new SCAR markers and chromosome walking, we eventually identified the candidate gene and characterized the gene by complementary transformation of the corresponding mutant in Arabidopsis. The functional copy of the candidate gene recovered the phenotype of the Arabidopsis mutant and the non-functional copy in B. rapa mutant did not so the candidate was confirmed to underpin the yellow-seeded mutation in B. rapa.

Similarly, we used SRAP to tag other genes controlling the seed-coat color trait in yellow sarson, another yellow-seeded B. rapa and also in yellow-seeded B. napus canola (Rahman et 
al., 2007, 2010). Yellow sarson is bright yellow-seeded and there is no color variation under different environments while all yellow-seeded canola accessions developed with yellow sarson are not pure yellow and the seed coat color varies due to differences in maturity and environments. Using SRAP screening, we identified several SRAP markers linked to one seed coat color gene. After extended flanking regions were sequenced, one closely-linked SRAP marker was successfully converted into SNP and SCAR markers. Meanwhile, we analyzed a yellow-seeded canola line that was developed with yellow sarson and found that three genes controlled the yellow-seeded color in this B. napus line (Rahman et al., 2010). We identified one SRAP marker that was linked closely to one seed color locus and confirmed that this locus was located on linkage group N9 of our ultradense genetic map of B. napus. The second locus was mapped on linkage group N13 of our ultradense genetic map. To identify SRAP markers for the third locus, we screened 768 SRAP primer combinations and eventually found one SRAP that was linked closely to the yellow seeded color locus. These SRAP, SNP and SCAR markers can be used in marker assisted selection of yellow-seeded trait in oilseed crops of B. rapa and B. napus.

SRAP technology is an effective molecular marker system to analyze qualitative and quantitative resistance to plant diseases. In general, qualitative and quantitative resistances are conferred by oligogenic or multigenic loci, respectively. In canola, blackleg is a major disease and qualitative resistance is available. We used the previously described ultradense genetic map to tag resistance genes to blackleg in B. napus. After screening 384 SRAP primer combinations, we identified two SRAP markers that were linked to a blackleg resistance gene. By compared the linked SRAP markers with the molecular markers on the ultradense B. napus genetic map that was constructed with another mapping population, we found that one SRAP marker corresponded to a SRAP marker on N10. Therefore we took the flanking SRAP markers of the mapped resistance locus on N10 and identified other SRAP markers on the genetic map that were also polymorphic in the mapping population of the blackleg resistance gene. Eventually, further analysis allowed us to identify two blackleg resistance genes in the region where one resistance gene was suggested by other researchers (Long et al., 2010).

In several reports, SRAP markers were used to map genes controlling resistance to plant diseases in several crop species. For instance, Yi et al., (2008) used SRAP, STS and SSR markers to tag a resistance gene $(P m 4 b)$ to powdery mildew in wheat. They tested 240 SRAP primer combinations and identified two SRAP markers linked to the $P m 4 b$ gene. Eventually, they mapped the Pm $4 b$ gene on chromosome 2AL that was flanked by SRAP, STS and SSR markers. In another study on gene mapping in wheat, Chen et al., (2012) used SSR, SRAP and TRAP markers to tag a wheat strip rust resistance gene. Using 400 SSR, 315 pairs of SRAP primers, and 40 pairs of TRAP primers to screen $\mathrm{F}_{1}, \mathrm{~F}_{2}$ and $\mathrm{BC}_{1}$ mapping populations, they constructed a fine map flanking the resistance gene locus on chromosome arm 2AS and suggested that the mapped resistance gene should be a novel one.

In rice, Zhao et al., (2010) searched for SSR markers linked to a dominant resistance gene (RSV1) to rice stripe virus and then used the SRAP method to find closely linked markers. They located RSV1 into a region flanked by SSR and SRAP markers. In maize, a new domi- 
nant resistance gene to maize head smut was tagged by SSR-BSA and SRAP-BSA methods (Li et al., 2012). Closely linked molecular markers were identified and used to transfer the resistance gene from the resistant source to elite lines via marker assisted selection to breed head smut resistant hybrid cultivars in maize.

In eggplant, Mutlu et al., (2008) tagged a Fusarium wilt resistance gene using SRAP, SRAPRGA, RAPD, and SCAR markers. They used 2316 primer combinations to identify molecular markers linked to the resistance gene, of which two SRAP markers were closely linked to the resistance gene. The SRAP markers were converted into SCAR markers and used in marker assisted selection of the Fusarium wilt resistance in eggplant.

Besides plant disease resistance, genes underpinning other traits have been tagged using SRAP technology, For instance, genes controlling two important traits, sex determination and tuberculate fruit in cucumber were tagged using SRAP technology (Li et al., 2008; Zhang et al., 2010a). In cucumber, there are three major gene loci, F/f, M/m, and A/a that determine various sex types. Li et al., (2008) analyzed M/m gene locus and identified 8 SRAP markers linked to this gene locus. Additionally, they used SRAP markers to perform chromosome walking and converted some SRAP markers to co-dominant SCAR markers through sequencing SRAP fragments. Eventually they identified very closely linked SRAP markers at a genetic distance of less than one cM. Similarly, Zhang et al., (2010a) performed gene tagging of cucumber tuberculate fruit. They found that the tuberculate fruit $(\mathrm{Tu})$ was controlled by a dominant gene and used a BSA strategy to identify molecular markers linked to this dominant gene. After testing 736 SRAP primer combinations, they found 9 SRAP markers that were linked to the Tu gene and used SSR markers to anchor this gene on chromosome 5, further indicating that they would use the mapping results to clone the Tu gene later.

Male sterility is a commonly used method to produce hybrid seeds for exploiting heterosis in crops. Since genic male sterility is usually controlled by a few genes, SRAP technology is useful to tag the genes underpinning male sterility. For example, Zhang et al., (2011c) used SRAP and SSR markers to tag a dominant genic male sterile gene in B. oleracea. They performed BSA analysis with SRAP and SSR markers. By screening polymorphisms between fertile bulks and sterile bulks with 26,417 SRAP primer pairs, they identified 14 SRAP markers that were linked to the male sterility gene MS-cd1. After sequencing the SRAP fragments, three SRAP markers were converted into SCAR markers that were very closely linked to the MS-cd1 gene. Moreover, through comparative genomics with SRAP sequences, they identified a collinear region on chromosome A10 in B. rapa corresponding to a collinear genomic region of chromosome 5 in Arabidopsis, which could lead to cloning of this gene in the future.

SRAP technology has also been used to tag quantitative traits using the same approaches as described previously in qualitative traits. In alfalfa, Castonguay et al., (2010) used SRAP to identify polymorphic genetic loci that controlled superior tolerance to freezing. Through BSA analysis, they found four SRAP markers that were associated with freezing tolerance and the frequency of their occurrence reflected changes in response to selection. In another report, SRAP was used to tag a major QTL controlling cadmium accumulation in oat (Tan- 
huanpaa et al 2007). The concentration of toxic cadmium in oat grains is often over the accepted limit and must be reduced. SRAP, RAPD and retrotransposon-microsatellite amplified polymorphism (REMAP) markers were used to perform BSA analysis in an F2 population and four molecular markers were identified to be associated with cadmium concentration in oat grains. All these four markers were located on the same linkage group, suggesting that this mapped QTL had major effect on grain cadmium concentration in oat.

\section{Genetic diversity}

Genetic diversity analysis is necessary in plant breeding, plant systematics and evolution, plant pathology. SRAP is an adequate molecular marker system for genetic diversity analysis in plants and fungi. Since SRAP has many features such as simplicity, reliability, flexibility, detection of multiple loci and cost-effectiveness, which allows beginners and experienced people to perform SRAP routinely with limited facilities or in well-established genomics labs. Since genome sequence information is not necessary for SRAP detection, SRAP can be used to perform genetic diversity analysis in a wide range of living organisms. We first used SRAP to analyze the genetic diversity of parental lines that were used to produce hybrid cultivars in B. napus (Riaz et al., 2001). As expected, we found that there was a positive correlation of genetic distance and hybrid performance. In celery, we used SRAP to tag a major resistance locus to celery mosaic virus (Ruiz et al., 2001).

In melons, Ferriol et al., (200) used SRAP and AFLP to analyze 69 accessions selected from morphotypes and unclassified types that belong to two subspecies, Cucurbita pepo ssp. pepo and ssp. ovifera. Among these accessions, some commercial cultivars and Spanish landraces represent diversified types in Europe. Their results showed that SRAP markers characterized well the morphological variability and the evolutionary history of these tested accessions better than AFLP markers. Molecular markers were used to identify new types for the development of new cultivars. The genetic diversity in the landraces of $C$. pepo spp. ovifera was detected with molecular markers, which is useful for preserving the diversity in this species.

In grasses, Budak et al., (2004a; 2004b; 2005) used SRAP to analyze genetic diversity and ploidy complexity in buffalograss. They found that SRAP markers were abundant and that they could distinguish genetic diversity among closely related cultivars. Their data showed that among several molecular markers (SSRs, ISSRs, SRAPs, and RAPDs), SRAP estimated the highest mean genetic dissimilarities in buffalograss. Additionally, they used SRAP and other markers to analyze ploidy complex and geographic origin of the Buchloe dactyloides genome and identified a significant correlation between the ploidy levels such as diploid, tetraploid, pentaploid, and hexaploid and the numbers of alleles detected using nuclear DNA markers. SRAP again was the best one among three molecular markers (ISSR, SSR, and SRAP, $\mathrm{r}=0.39,0.39$, and 0.41$)$.

Similarly, Gulsen et al., (2009) used SRAP, peroxidase gene polymorphism (POGP), ISSR and RAPD to study the relationship of ploidy levels, geographic locations and genetic di- 
versity in bermudagrass. They found that there was a significant correlation between ploidy levels in diploids, triploids, tetraploids, pentaploids, and hexaploids and band frequencies of molecular markers $(r=0.62, \mathrm{P}<0.001)$, suggesting that ploidy levels resulted in genome variation and genetic diversity. Geographic locations of Cynodon accessions also contributed to genetic diversity based on molecular marker analysis. They suggested that combining several molecular markers would be more efficient to evaluate genetic diversity and genetic structure in bermudagrass and eventually broaden genetic basis for developing new cultivars.

In elephant grass, Xie et al., (2009) used SRAP markers to study the genetic diversity and relationships of commonly used cultivars in China. They generated 1,395 genetic loci with 62 SRAP primer combinations with an average of 22.5 genetic loci per primer combination. They found that SRAP loci were very polymorphic $(72.8 \%)$ and used these SRAPs to estimate the genetic diversity within and between elephant grass cultivars. The results showed the genetic diversity within cultivars was less than that among tested cultivars and the relationship of those tested cultivars was also estimated.

In cereal crops, Zaefizadeh and Goleiv (2009) analyzed genetic diversity and relationships among durum wheat landraces by SRAP marker and phenotypic differences. They used 65 SRAP markers and 27 traits to perform cluster analysis of 40 subconvars of Triticum durum landraces from the region of North West Iran and Azerbaijan. Traits failed to detect any geographic association in durum landraces while 12 combinations of SRAP markers were distinguishable among these landraces, suggesting that SRAP technology is useful for genetic diversity and evolutionary relationship analysis, marker assisted selection and genetic map construction in durum wheat. Yang et al., (2010) used SRAP markers to analyze the genetic diversity of hulless barley cultivars from Sichuan, Gansu, Tibet, Qinghai and Yunnan provinces of the Qinghai-Tibet Plateau in China. With 20 SRAP primer combinations, they detected 153 polymorphic loci and used these SRAP markers to classify 68 hulless barley accessions into four major groups using a unweighted pair-grouping method with arithmetic averages (UPGMA) analysis. They concluded that SRAP was an effective method to perform genetic diversity in hulless barley and develop new cultivars.

In rice, Dai et al., (2012) developed indica- and japonica-specific markers using SRAP, TRAP, and SSR markers and performed genetic diversity analysis of Asian Oryza sativa varieties. In general, rice varieties are classified into O. sativa ssp. japonica kata and ssp. indica kata. In this report, they used 45 rice varieties in a cultivated and wild rice collection to study the genetic diversity in rice. By developing 90 indica- and japonica-specific genetic loci, they could easily distinguish typical indica and japonica subspecies and determined whether a domesticated rice variety came from the indica or japonica type.

In alfalfa, Vandemark et al.,(2006) used SRAP markers to analyze genetic relationships among historical sources of alfalfa germplasm in North American. Their results showed that SRAP detected highly polymorphic loci $(>90 \%)$ in alfalfa, which distinguished nine original sources of Medicago germplasm based on genetic similarity calculated with SRAP markers. They suggested that SRAP technology is an adequate marker system for detecting polymorphisms in alfalfa. 
In sesame, Zhang et al., (2010b) performed genetic diversity analysis using SRAP and SSR markers. They analyzed 404 landraces from a sesame collection in China. Using11 SRAP and 3 SSR markers, they produced 175 fragments, of which 126 were polymorphic with an average polymorphism rate of $72 \%$. They calculated several parameters such as Jaccard's genetic similarity coefficients, Nei's gene diversity and Shannon's information index and constructed a dendrogram with all the 404 landraces. According to the dendrogram, landraces from different agro-ecological zones did not cluster together, suggesting that geographical locations did not represent the greater genetic variation among the sesame landraces. They concluded that SRAP and SSR markers would be useful to study sesame genetic diversity and understand the relationship of those indigenous landraces, which would guide the collection, protection and utilization of sesame landraces in breeding purposes.

In banana and plantain, Youssef et al., (2011) used SRAP and AFLP markers to analyze 40 Musa accessions including commercial cultivars and wild species. They developed 353 SRAP and 787 AFLP markers to perform cluster analysis using an unweighted pair-grouping method with arithmetic averages (UPGMA) and principal coordinate (PCO) analysis. They eventually assigned all the 40 accessions into corresponding Eumusa, Australimusa, Callimusa and Rhodochlamys sections and species. They found that SRAP and AFLP polymorphism amongst sections and species and the relationships within Eumusa species and subspecies were not consistent and suggested that SRAP produced threefold more specific and unique loci than AFLP. Therefore, the data showed that SRAP markers were effective to distinguish M. acuminata, M. balbisiana and M. schizocarpa in the Eumusa section, and also triploid plantains and cooking bananas.

In grape, Guo et al., (2012) used SRAP markers to study genetic variability and relationships of cultivated wine-type Vitis vinifera and wild Vitis species. They selected 76 grape genotypes representing indigenous and new varieties and wild Vitis species from China and other countries. After testing 100 SRAP primer combinations, they selected 19 primer combinations based on primer performance to produce 228 genetic loci, of which $78.63 \%$ were polymorphic with an average polymorphism information content value of 0.76 . The SRAP markers were used to perform cluster analysis to evaluate Nei and Li's similarity coefficients by unweighted pair-group method of arithmetic averages (UPGMA) analysis. Additionally, they performed principal coordinate analysis (PCoA) to plot all 76 grape genotypes which showed a similar cluster pattern to that in the dendrogram, representing their geographical origins and taxonomic classification of these grape varieties. All the results indicated that three main groups including table grape of $V$. vinifera, table grape of EuroAmerica hybrids and wine grape of $V$. vinifera, wild Vitis species were identified and also the table $V$. vinifera group was genetically different from the wine-type $V$. vinifera and wild Vitis species originated from America and China. So they suggested that SRAP markers are informative and grape germplasm in China contains abundant genetic diversity.

In medicinal plants, Ortega et al., (2007) analyzed genetic diversity of cultivated and non-cultivated mashua, Tropaeolum tuberosum that were grown in six communities in the Cusco region of Perú and selected from the germplasm collection at the International Potato Center (CIP) using SRAP markers. Mashua is used as a medicinal plant, possibly 
due to a high concentration of glucosinolates in mashua roots. DNA fingerprinting generated by SRAP markers showed that mashua is a genetically variable crop. The genetic analysis also showed that most non-cultivated accessions were likely feral races resulting from escape from cultivation rather than wild relatives. In another medicinal plant, Wang et al., (2012) analyzed the genetic diversity of 35 wild goat's rue accessions (Galega officinalis L.) collected from Russia and Europe countries using ISSR and SRAP markers. Although there was some discrepancy between ISSR and SRAP markers, the clustering patterns of genotypes were relatively consistent between these two kinds of molecular markers in this study. They indicated that both markers were useful for goat's rue germplasm characterization, improvement, and conservation.

In ornamental plants, Hao et al., (2008) used SRAP technology to perform genetic diversity analysis of 29 ornamental and medicinal Paeonia. Dendrogram and principle component analysis indicated that SRAP markers well characterized the genetic relationships of these 29 peony cultivars, which is useful to guide parent selection and molecular marker assisted selection in Paeonia breeding. In another ornamental plant, Feng et al., (2009b) performed genetic analysis of diversity and population structure of Celosia argentea and related species using SRAP markers. They included 16 populations of $C$. argentea. and 6 populations of $C$. cristata. from China. Using 10 SRAP primer combinations, they produced 507 scored bands, of which 274 were polymorphic. With UPGMA cluster analysis, they constructed a phylogenetic tree and calculated genetic distances of all 22 populations. The results showed that the genetic distances of all populations were coincident with their geographic origins. Additionally, they identified one SRAP marker separating accessions in C. argentea from those in C. cristata and suggested that the extensive genetic diversity in C. argentea populations would be very useful for breeding and conservation of $C$. argentea varieties in the future.

In chrysanthemum, Zhang et al., (2011a) did a genetic diversity study on two flowering traits of chrysanthemum, initial blooming time and the duration of flowering. They identified two pairs of major genes with high levels of inheritance. Using SRAP technology, they performed association mapping of these two traits and identified SRAP markers that were significantly associated with phenotypes, suggesting that SRAP markers might be useful in chrysanthemum breeding. In another report on ornamental plants, Soleimani et al., (2012) used wild, cultivated, and ornamental pomegranates (Punica granatum L.) in Iran to perform analysis of genetic diversity and population structure with SRAP molecular markers. They produced 133 SRAP markers with 13 SRAP primer combinations to evaluate the genetic diversity of 63 pomegranate genotypes from five different geographical regions of Iran. Their data showed that the average polymorphism information content value was 0.28 and the genetic distance was 0.10 to 0.37 with an average of 0.24 in all 63 genotypes. Cluster analysis allowed them to identify the relationship between ornamental and wild genotypes. They found that the genetic variation of genotypes from various regions was bigger than that of intra regions. They concluded that SRAP markers could be an effective marker system in the analysis of genetic diversity and population structure in pomegranate.

In woody plants, Li et al., (2010) did genetic diversity analysis of sea buckthorn which is grown as a nutritious berry crop. They produced 191 polymorphic loci using SRAP technol- 
ogy to perform cluster analysis of 77 accessions, of which 73 Hippophae rhamnoides were classified into 2 groups and $4 \mathrm{H}$. salicifolia, into 1 group. They associated SRAP markers with dried-shrink disease (DSD) resistance and suggested SRAP markers are useful for breeding new sea buckthorn lines with resistance to DSD. Feng et al., (2009a) reported on the genetic diversity analysis of Pinus koraiensis using SRAP markers. They obtained 24 to 33 loci per primer combination and used 143 SRAP markers to analyze 480 samples collected from 24 provinces in China. They found that there was no significant difference in genetic diversity among provinces. However, genetic variation of intra population accounted for $93.355 \%$ of the total variation.

In fungi, Sun et al., (2006) used SRAP markers to classify Ganoderma lucidum strains. They performed genetic diversity analysis with 31 accessions collected from several countries. Using 75 polymorphic loci, they classified all 31 accessions into five groups. The results showed that G. lucidum strains were significantly different from G. sinense and G. lucidum in China, also different from G. lucidum in Yugoslavia. They suggested that SRAP markers are useful in taxonomy and systematics of Ganoderma strains within basidiomycetes. In another fungus, Tang et al., (2010) analyzed Chinese Auricularia auricula strains using SRAP and ISSR markers. They found both SRAP and ISSR markers were abundant in A. auricula and could be used to effectively distinguish all tested strains. After phylogenetic analysis, they classified $34 \mathrm{~A}$. auricula strains into four or five major groups using the UPGMA method. They suggested that genetic diversity information would be used in A. auricula breeding programs to develop new medicinal mushroom. Fu et al., (2010) performed genetic diversity analysis in 23 elite Lentinula edodes strains from China using RAPD, ISSR and SRAP markers. In total, they used 16 RAPD primers, 5 ISSR primers and 23 SRAP primer combinations to produce 138, 77 and 144 bands, respectively. After UPGMA clustering analysis, they classified all 23 L. edodes strains into three or four groups. However, all groups showed high levels of similarity, showing a low level of genetic diversity in all tested strains.

\section{Other applications}

SRAP amplification is actually a small portion of all possible sampling of a genome. So SRAP can be used to produce a reduced genome samples when multiple SRAP reactions are pooled. As described previously, pooled SRAP produces can be directly sequenced using next generation sequencing technologies. When replacing genomic DNA with cDNA samples, SRAP is adequate to perform gene expression profiling and also construct cDNA genetic maps.

More recently, Yu et al., (2012) used SRAP markers to distinguish fertile somatic hybrids of G. hirsutum L. and G. trilobum produced by protoplast fusion. They obtained fertile somatic hybrids by symmetric electrofusion of protoplasts of tetraploid upland cotton G. hirsutum and wild cotton G. trilobum. These hybrids were confirmed using morphological characteristics, flow cytometric analysis, and molecular markers including RAPD, SRAP and AFLP. 
In aquaculture, Ding et al., (2010) used SRAP and SCAR markers to differentiate two cultured populations in grass carp (Ctenopharyngodon idella). Through cloning and sequencing SRAP fragments, they developed SCAR markers to characterize individuals from the cultured population and the wild population, showing different frequencies of SCAR alleles (87\% in the cultured population and $6 \%$ in the wild), suggesting that this SCAR might serve as a specific molecular marker for the cultured population. They also identified eight SRAP fragments that shared high similarities to functional genes.

\section{Summary remarks}

SRAP was first used to construct a genetic map and tag genes in Brassica oleracea in 2001 (Li and Quiros, 2001). This molecular marker technology is simply performed with one round PCR to amplify multiple or occasionally over a hundred loci in a genome. In its PCR reaction mixture, two random primers are included, which leads to maximum flexibility in primer designing and primer labelling. There is no limitation on primer combinations and one labelled primer may be combined with any number of unlabelled primers. Most SRAP products fall into a size range of 100 to 1000 base pairs, which can be separated in both polyacrylamide and agarose gels. In automatized detection, one SRAP primer is fluorescently labelled and SRAP products can be analyzed using advanced instruments such as an ABI genetic analyzer, which dramatically increases throughput of SRAP molecular marker detection.

There is a wide range of applications of SRAP technology such as genetic map construction, genetic diversity analysis, gene tagging and cloning. Since SRAP detects multiple loci in one reaction, it is feasible to construct ultradense genetic maps with over 10,000 SRAP molecular markers. SRAP has advantages over other molecular detection techniques in gene tagging and cloning and allows screening thousands of loci shortly to pinpoint the genetic position underlying the trait of interest. Sequencing SRAP products enhances the applications of SRAP technology. In well characterized genomes, SRAP sequences are used to identify the chromosomal region of mapped genes while in species without a known whole genome sequence, sequences of SRAP markers on a genetic map allow arranging sequence contigs and assembly of a whole genome sequence.

SRAP molecular technology is very useful in plant breeding. In QTL mapping, common QTL for the same trait of interest can be effectively identified. Since SRAP has a high throughput feature, multiple mapping populations can be analyzed effectively to construct several genetic maps. In addition, the same set of SRAP primers allows detection of the same genetic loci, which can used to align several genetic maps. SRAP is effective and efficient in marker assisted selection in plant breeding since thousands of samples can be analyzed inexpensively. SRAP technology has been commonly used in analysis of genetic diversity of many plant species. Currently, SRAP are used in most crops, tree species, ornamental and medicinal plants. 


\section{Author details}

Genyi Li ${ }^{1}$, Peter B. E. McVetty ${ }^{1}$ and Carlos F. Quiros ${ }^{2}$

1 Department of Plant Science, University of Manitoba, MB, Canada

2 Department of Plant Sciences, University of California, Davis, CA, USA

\section{References}

[1] Budak, H. R. C., Shearman, I., Parmaksiz, R. E., Gaussoin, T. P., \& Riosdan, D. (2004a). Molecular characterization of Buffalograss germplasm using sequence-related amplified polymorphism markers. Theor Appl Genet. , 108, 328-334.

[2] Budak, H., Shearman, R. C., Parmaksiz, I., \& Dweikat, I. (2004b). Comparative analysis of seeded and vegetative biotype buffalograsses based on phylogenetic relationship using ISSRs, SSRs, RAPDs, and SRAPs. Theor Appl Genet. , 109, 280-8.

[3] Budak, H., Shearman, R. C., Gulsen, O., \& Dweikat, I. (2005). Understanding ploidy complex and geographic origin of the Buchloe dactyloides genome using cytoplasmic and nuclear marker systems. Theor Appl Genet. , 111, 1545-52.

[4] Castonguay, Y., Cloutier, J., Bertrand, A., Michaud, R., \& Laberge, S. (2010). SRAP polymorphisms associated with superior freezing tolerance in alfalfa (Medicago sativa spp. sativa). Theor Appl Genet. , 120, 1611-9.

[5] Chen, G., Geng, J., Rahman, M., Liu, X., Tu, J., Fu, T., Li, G., Mc Vetty, P. B. E., \& Tahir, M. (2009). Identification of QTL for oil content, seed yield, and flowering time in oilseed rape (Brassica napus). Euphytica , 142, 161-174.

[6] Chen, M., Wei, C., Qi, J., Chen, X., Su, J., Li, A., Tao, A., \& Wu, W. (2011). Genetic linkage map construction for kenaf using SRAP, ISSR and RAPD markers. Plant Breed. , 130, 679-687.

[7] Chen, S., Chen, G., Chen, H., Wei, Y., Li, W., Liu, Y., Liu, D., Lan, X., \& Zheng, Y. (2012). Mapping stripe rust resistance gene YrSph derived from Tritium sphaerococcum Perc. with SSR, SRAP, and TRAP markers. Euphytica , 185, 19-26.

[8] Chen, W., Zhang, Y., Liu, X., Chen, B., Tu, J., \& Fu, T. (2007). Detection of QTL for six yield-related traits in oilseed rape (Brassica napus) using DH and immortalized $\mathrm{F}(2)$ populations. Theor Appl Genet. , 115, 849-58.

[9] Chen, X., Xia, Z., Fu, Y., Lu, C., \& Wang, W. (2010). Constructing a genetic linkage map using an $\mathrm{F}_{1}$ population of non-inbred parents in cassava (Manihot esculenta Crantz). Plant Molecular Biology Reporter , 28, 676-683. 
[10] Dai, X., Yang, Y., Zhou, L., Ou, L., Liang, M., Li, W., Kang, G., \& Chen, B. (2012). Analysis of indica- and japonica-specific markers of Oryza sativa and their applications. Plant Systematics and Evolution, 298, 287-296.

[11] Ding, W., Cao, Z., \& Cao, L. (2010). Molecular analysis of grass carp (Ctenopharyngodon idella) by SRAP and SCAR molecular markers. Aquaculture International , 18, 575-587.

[12] Feng, F., Chen, M., Zhang, D., Sui, X., \& Han, S. (2009a). Application of SRAP in the genetic diversity of Pinus koraiensis of different provenances. African Journal of Biotechnology , 8, 1000-1008.

[13] Feng, N., Xue, Q., Guo, Q., Zhao, R., \& Guo, M. (2009b). Genetic diversity and population structure of Celosia argentea and related species revealed by SRAP. Biochem Genet. , 47, 521-32.

[14] Ferriol, M., Pico, B., \& Nuez, F. (2003). Genetic diversity of a germplasm collection of Cucurbita pepo using SRAP and AFLP markers. Theor Appl Genet. , 107, 271-282.

[15] Fu, F. Y., Liu, L. Z., Chai, Y., Chen, L., Yang, T., Jin, M., , A., Yan, X., Zhang, Z., \& Li, J. (2007). localization of QTLs for seed color using recombinant inbred lines of Brassica napus in different environments. Genome, 50, 840-854.

[16] Fu, L., Zhang, H., Wu, X., Li, H., Wei, H., Wu, Q., \& Wang, L. (2010). Evaluation of genetic diversity in Lentinula edodes strains using RAPD, ISSR and SRAP markers. World Journal of Microbiology and Biotechnology , 26, 709-716.

[17] Gao, M., Li, G., Yang, B., Qiu, D., Farnham, M., \& Quiros, C. F. (2007). High-density Brassica oleracea map: Identification of useful new linkages. Theor Appl Genet. , 115, 277-287.

[18] Gulsen, O., Sever-Mutlu, S., Mutlu, N., Tuna, M., Karaguzel, O., Shearman, R. C., Riordan, T. P., \& Heng-Moss, T. M. (2009). Polyploidy creates higher diversity among Cynodon accessions as assessed by molecular markers. Theor Appl Genet. , 118, 1309-19.

[19] Gulsen, O., Uzun, A., Canan, I., Seday, U., \& Canihos, E. (2010). A new citrus linkage map based on SRAP, SSR, ISSR, POGP, RGA and RAPD markers. Euphytica, 173, 265-277.

[20] Guo, D., Zhang, J., Liu, C., Zhang, G., Li, M., \& Zhang, Q. (2012). Genetic variability and relationships between and within grape cultivated varieties and wild species based on SRAP markers. Tree Genetics \& Genomes , 8, 789-800.

[21] Hao, Q., Liu, Z., Shu, Q., Zhang, R., Rick, J. D., \& Wang, L. (2008). Studies on Paeonia cultivars and hybrids identification based on SRAP analysis. Hereditas , 145, 38-47.

[22] Jones, N., Ougham, H., Thomas, H., \& Pasakinskiene, I. (2009). Markers and mapping revisited: finding your gene. New Phytol. , 183, 935-66. 
[23] Levi, A., Thomas, C. E., Trebitsh, T., Salman, A., King, J., Karalius, J., Newman, M., Reddy, O. U. K., Xu, Y., \& Zhang, X. (2006). An extended linkage map for watermelon based on SRAP, AFLP, SSR, ISSR, and RAPD markers. J Am Soc Hortic Sci. , 131, 393-402.

[24] Li, G., Gao, M., Yang, B., \& Quiros, C. F. (2003). Gene to gene alignment between the Arabidopsis and Brassica oleracea genomes. Theor Appl Genet. , 107, 168-80.

[25] Li, G., \& Quiros, C. F. (2001). Sequence-related amplified polymorphism (SRAP), a new marker system based on a simple PCR reaction: Its application to mapping and gene tagging in Brassica. Theor Appl Genet. , 103, 455-461.

[26] Li, H., Ruan, C. J., Silva, T., Jaime, A., \& Liu, B. Q. (2010). Associations of SRAP markers with dried-shrink disease resistance in a germplasm collection of sea buckthorn (Hippophae rhamnoides L.). Genome , 53, 447-457.

[27] Li, W., Xu, X., Li, G., Guo, Q., Wu, S., Jiang, Y., Dong, H., Weng, M., Jin, D., Wu, J., $\mathrm{Ru}, \mathrm{Z}$. , \& Wang, B. (2012). Characterization and molecular mapping of RsrR, a resistant gene to maize head smut. Euphytica (DOIs10681-012-0747-4, in press).

[28] Li, W., Zhang, J., Mou, Y., Geng, J., Mc Vetty, P. B. E., Hu, S., \& Li, G. (2011). Integration of Solexa sequences on an ultradense genetic map in Brassica rapa L. BMC Genomics 12: 249.

[29] Li, Z., Pan, J., Guan, Y., Tao, Q., He, H., Si, L., \& Cai, R. (2008). Development and fine mapping of three co-dominant SCAR markers linked to the $\mathrm{M} / \mathrm{m}$ gene in the cucumber plant (Cucumis sativus L.).Theor Appl Genet. , 117, 1253-60.

[30] Lin, Z.., He, D., Zhang, X., Nie, Y., Guo, X., Feng, C., et al. (2005). Linkage map construction and mapping QTL for cotton fiber quality using SRAP, SSR and RAPD. Plant Breed. , 124, 180-187.

[31] Liu, L., , X., Wei, J., Qin, J., \& Mo, C. (2011). The first genetic linkage map of Luohanguo (Siraitia grosvenorii) based on ISSR and SRAP markers. Genome , 54, 19-25.

[32] Long, Y., Wang, Z., Sun, Z., Fernando, D. W. G., Mc Vetty, P. B. E., \& Li, G. (2010). Identification of two blackleg resistance genes and fine mapping of one of these two genes in a Brassica napus canola cultivar 'Surpass 400'. Theor Appl Genet. , 122, 1223-1231.

[33] Mutlu, N., Boyaci, F. H., Göçmen, M., \& Abak, K. (2008). Development of SRAP, SRAP-RGA, RAPD and SCAR markers linked with a Fusarium wilt resistance gene in eggplant. Theor Appl Genet. , 117, 1303-12.

[34] Ortega, O., Duran, E., Arbizu, C., Ortega, R., Roca, W., Potter, D., \& Quiros, C. F. (2007). Pattern of genetic diversity of cultivated and non-cultivated mashua, Tropaeolum tuberosum, in the Cusco region of Perú. Genetic Resources and Crop Evolution, 54, 807-821. 
[35] Rahman, M., Li, G., Schroeder, D., \& Mc Vetty, P. B. E. (2010). Inheritance of seed coat color genes in Brassica napus (L.) and tagging the genes using SRAP, SCAR and SNP molecular markers. Molecular Breed. , 26, 439-453.

[36] Rahman, M., Sun, Z., Mc Vetty, P. B. E., \& Li, G. (2007). Development of SRAP, SNP and SCAR molecular markers for the major seed coat color gene in Brassica rapa. Theor Appl Genet. , 115, 1101-7.

[37] Riaz, A., Li, G., Quresh, Z., Swati, M., \& Quiros, C. F. (2001). Genetic diversity of oilseed Brassica napus inbred lines based on sequence-related amplified polymorphism and its relation to hybrid performance. Plant Breed. , 120, 1-5.

[38] Ruiz, J. J., Pico, B., Li, G., D’Antonio, V., Falk, B., \& Quiros, C. F. (2001). Identification of markers linked to a Celery Mosaic Virus resistance gene in celery. J Amer Soc for Hortic Sci. , 126(4), 432-435.

[39] Soleimani, M. H., Talebi, M., \& Sayed-Tabatabaei, B. E. (2012). Use of SRAP markers to assess genetic diversity and population structure of wild, cultivated, and ornamental pomegranates (Punica granatum L.) in different regions of Iran. Plant Systematics and Evolution, 298, 1141-1149.

[40] Sun, S., Gao, W., Lin, S., Zhu, J., Xie, B., \& Lin, Z. (2006). Analysis of genetic diversity in Ganoderma population with a novel molecular marker SRAP. Applied Microbiology and Biotechnology, 72, 537-543.

[41] Sun, Z., Wang, Z., Tu, J., Zhang, J., Yu, F., Mc Vetty, P. B. E., \& Li, G. (2007). An ultradense genetic recombination map for Brassica napus, consisting of 13551 SRAP markers. Theor Appl Genet. , 114(8), 1305-17.

[42] Tang, L., Xiao, Y., Li, L., Guo, Q., \& Bian, Y. (2010). Analysis of genetic diversity among Chinese Auricularia auricula cultivars using combined ISSR and SRAP markers. Current Microbiology , 61, 132-140.

[43] Tanhuanpää, P., Kalendar, R., Schulman, A. H., \& Kiviharju, E. (2007). A major gene for grain cadmium accumulation in oat (Avena sativa L.). Genome , 50, 588-94.

[44] Vandemark, G. J., Ariss, J. J., Bauchan, G. A., Larsen, R. C., \& Hughes, T. J. (2006). Estimating genetic relationships among historical sources of alfalfa germplasm and selected cultivars with sequence related amplified polymorphisms. Euphytica , 152, 9-16.

[45] Wang, Z., Wang, J., Wang, X., Gao, H., Dzyubenko, N. I., \& Chapurin, V. F. (2012). Assessment of genetic diversity in Galega officinalis L. using ISSR and SRAP markers. Genetic Resources and Crop Evolution , 59, 865-873.

[46] Xie, W., Zhang, X., Cai, H., Huang, L., Peng, Y., \& , X. (2011). Genetic maps of SSR and SRAP markers in diploid orchardgrass (Dactylis glomerata L.) using the pseudotestcross strategy. Genome , 54, 212-221. 
[47] Xie, X., Zhou, F., Zhang, X., \& Zhang, J. (2009). Genetic variability and relationship between MT-1 elephant grass and closely related cultivars assessed by SRAP markers. Journal of Genetics , 88, 281-290.

[48] Xu, L., Wang, L., Gong, Y., Dai, W., Wang, Y., Zhu, X., Wen, T., \& Liu, L. (2012). Genetic linkage map construction and QTL mapping of cadmium accumulation in radish (Raphanus sativus L.).Theor Appl Genet. , 125, 659-70.

[49] Xue, D., Feng, S., Zhao, H., Jiang, H., Shen, B., Shi, N., Lu, J., Liu, J., \& Wang, H. (2010). The linkage maps of Dendrobium species based on RAPD and SRAP markers. Journal of Genetics and Genomics , 37, 197-204.

[50] Yang, P., Liu, X., Liu, X., Yang, W., \& Feng, Z. (2010). Diversity analysis of the developed qingke (hulless barley) cultivars representing different growing regions of the Qinghai-Tibet Plateau in China using sequence related amplified polymorphism (SRAP) markers. African Journal of Biotechnology , 9, 8530-8538.

[51] Yeboah, M. A., Xuehao, C., Feng, C., Liang, G., \& Gu, M. (2007). A genetic linkage map of cucumber (Cucumis sativus L.) combining SRAP and ISSR markers. African Journal of Biotechnology , 6, 2784-2791.

[52] Yi, Y., Liu, H., Huang, X., An, L., Wang, F., \& Wang, X. (2008). Development of molecular markers linked to the wheat powdery mildew resistance gene $P m 4 b$ and marker validation for molecular breeding. Plant Breed. , 127, 116-120.

[53] Youssef, M., James, A. C., Rivera-Madrid, R., Ortiz, R., \& Escobedo-Gracia, Medrano. R. M. (2011). Musa genetic diversity revealed by SRAP and AFLP. Molecular Biotechnology , 47, 189-199.

[54] Yu, J., Yu, S., Lu, C., Wang, W., Fan, S., Song, M., Lin, Z., Zhang, X., \& Zhang, J. (2007). High-density linkage map of cultivated allotetraploid cotton based on SSR, TRAP, SRAP and AFLP markers. Journal of Integrative Plant Biology , 49, 716-724.

[55] Yu, X., Chu, B., Liu, R., Sun, J., Brian, J. J., Wang, H., Shuijin, Z., \& Sun, Y. (2012). Characteristics of fertile somatic hybrids of G. hirsutum L. and G. trilobum generated via protoplast fusion. Theor Appl Genet. 2012 Jul 10.

[56] Zaefizadeh, M., \& Goleiv, R. (2009). Diversity and relationships among durum wheat landraces (subconvars) by SRAP and phenotypic marker polymorphism. Research Journal of Biological Sciences , 4, 960-966.

[57] Zhang, F., Chen, S., Chen, F., Fang, W., Deng, Y., Chang, Q., \& Liu, P. (2011a). Genetic analysis and associated SRAP markers for flowering traits of chrysanthemum (Chrysanthemum morifolium). Euphytica , 177, 15-24.

[58] Zhang, F., Chen, S., Chen, F., Fang, W., Chen, Y., \& Li, F. (2011b). SRAP-based mapping and QTL detection for inflorescence-related traits in chrysanthemum (Dendranthema morifolium). Molecular Breed. 27: 11-23, 
[59] Zhang, J., Lu, Y., Yuan, Y., Zhang, X., Geng, J., Chen, Y., Cloutier, S., Mc Vetty, P. B. E., \& Li, G. (2009a). Map-based cloning and characterization of a gene controlling hairiness and seed coat color traits in Brassica rapa. Plant Molecular Biol. , 69, 553-563.

[60] Zhang, W., He, H., Guan, Y., Du, H., Yuan, L., Li, Z., Yao, D., Pan, J., \& Cai, R. (2010a). Identification and mapping of molecular markers linked to the tuberculate fruit gene in the cucumber (Cucumis sativus L.). Theor Appl Genet. , 120, 645-654.

[61] Zhang, W., Pan, J., He, H., Zhang, C., Li, Z., Zhao, J., Yuan, X., Zhu, L., Huang, S., \& Cai, R. (2012). Construction of a high density integrated genetic map for cucumber (Cucumis satious L.) Theor Appl Genet. , 124, 249-259.

[62] Zhang, Y., Zhang, X., Hua, W., Wang, L., \& , Z. (2010b). Analysis of genetic diversity among indigenous landraces from sesame (Sesamum indicum L.) core collection in China as revealed by SRAP and SSR markers. Genes E Genomics , 32, 207-215.

[63] Zhang, X., Wu, J., Zhang, H., , Y., Guo, A., \& Wang, X. (2011c). Fine mapping of a male sterility gene MS-cd1 in Brassica oleracea. Theor Appl Genet. , 123, 231-8.

[64] Zhang, Z., Hu, M., Zhang, J., Liu, D., Zheng, J., Zhang, K., Wang, W., \& Wan, Q. (2009b). Construction of a comprehensive PCR-based marker linkage map and QTL mapping for fiber quality traits in upland cotton (Gossypium hirsutum L.) Molecular Breed. , 24, 49-61.

[65] Zhao, F., Cai, Z., Hu, T., Yao, H., Wang, L., Dong, N., Wang, B., Ru, Z., \& Zhai, W. . (2010). Genetic analysis and molecular mapping of a novel gene conferring resistance to rice stripe virus. Plant Molecular Biology Reporter , 28, 512-518. 
